\title{
Prognostic significance of GRP78/BiP expression in patients with Stage III/IV hypopharyngeal squamous cell carcinoma
}

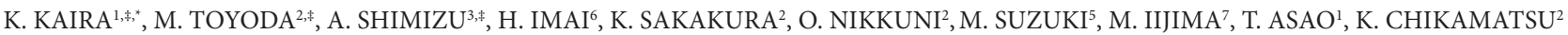 \\ ${ }^{1}$ Department of Oncology Clinical Development; ${ }^{2}$ Department of Otolaryngology-Head and Neck Surgery; ${ }^{3}$ Department of Dermatology; ${ }^{4}$ De- \\ partment of Diagnostic Pathology, Gunma Graduate School of Medicine; ${ }^{5}$ Department of Head \& Neck Surgery; ${ }^{6}$ Department of Respiratory \\ medicine; 'Department of Pathology and Clinical Laboratories, Gunma Prefectural Cancer Center \\ *Correspondence: kkaira1970@yahoo.co.jp \\ Contributed equally to this work.
}

Received October 2, 2015 / Accepted December 15, 2105

\begin{abstract}
The immunoglobulin heavy chain binding protein (BiP)/glucose-regulated protein 78 (GRP78) plays an essential role in the endoplasmic reticulum (ER) stress, and GRP78/BiP is known to be highly expressed in various human neoplasms. The clinicopathological features of GRP78/BiP expression in patients with advanced hypopharyngeal squamous cell carcinoma (HSCC) remain unclear. The aim of this study is to elucidate the prognostic significance of GRP78/BiP for HSCC.

A total of 68 patients with advanced HSCC (stage III/IV) were analyzed, and tumor specimens were stained with immunohistochemistry for GRP78/BiP, Ki-67, and microvessel density (MVD), as determined through CD34 and p53 levels.

GRP78/BiP was highly expressed in 80.8\% (55/68) of all patients. The expression level of GRP78/BiP disclosed no significant relationship with any variables. Multivariate analysis confirmed that low expression of GRP78/BiP was an independent prognostic factor for predicting poor overall survival and progression-free survival in patients with advanced HSCC.

The decreasing expression of GRP78/BiP was identified as a significant predictor related to shorter survival duration after surgery for advanced HSCC. Our study suggests that the reduced expression of GRP78/BiP contributes to worse survival for patients with advanced head and neck cancer.
\end{abstract}

Key words: GRP78/BiP, ER stress, hypopharynx, head and neck cancer, squamous cell carcinoma, immunohistochemistry

Head and neck cancer is a difficult disease to survive; improving survival advantage after systemic chemotherapy, especially in patients with advanced staging and cancers of the oral cavity, oropharynx, hypopharynx, and larynx, is difficult [1]. Of these head and neck cancers, hypopharyngeal squamous cell carcinoma (HSCC) is one with the worst prognosis regardless of appropriate therapeutics. HSCC is known to be a highly aggressive neoplasm, including invasion of the adjacent structures and enlarged cervical lymph node metastases. Currently, the efficacy of standard treatment against advanced HSCC is limited and an effective targeting therapeutic method is necessary to improve the outcome. Therefore, clinical and pathological biomarkers as significant predictors after treatment should be established to improve the prognosis of therapy.

The glucose-regulated protein GRP78, also referred to as an immunoglobulin heavy chain binding protein (BiP), is a $78 \mathrm{k}-\mathrm{Da}$ protein and a major molecular chaperone at the endoplasmic reticulum (ER). It has been elucidated in the cytoplasm and on the cell membrane [2,3]. GRP78/BiP is utilized in the folding and assembly of newly synthesized proteins in the ER and possesses enhanced resistance to ERstress-induced apoptosis [2.3]. It has been described that GRP78/BiP is highly expressed in many cancer cell lines and human cancers, correlated with malignant formation, metastases, and resistance to chemotherapy [3,4]. Recently, the clinicopathological investigations of GRP78/BiP in cancer patients such as lung cancer, breast cancer, hepatocellular cancer, prostate cancer, and gastric cancer, have been explored and discussed in several studies [3, 5-12]. In head and neck cancers, especially, there has been considerable focus on the malignant aggressiveness and prognostic significance of GRP78/BiP expression in patients with oral SCC [5-7]. These studies disclosed that the expression of GRP78/BiP is related 
to the survival and the tumor aggressiveness such as disease staging, metastasis, and tumor size [5-12]. One report has described that the positive GRP78/BiP expression was proved to be a significant independent biomarker for predicting poor prognosis of oral SCC, but another report documented that weak GRP78/BiP expression is more closely associated with advanced disease stage and lymph node metastasis than strong GRP78/BiP expression [5-8]. Presently it is unknown whether the patients with oral SCC show more unfavorable prognosis in the high expression of GRP78/BiP than in the low expression of GRP78/BiP. However, there is no report regarding the relationship between the expression level of GRP78/BiP and clinicopathological features in patients with HSCC. Based on these background, the aim of our study was to examine the clinicopathological significance of GRP78/BiP protein expression as an ER stress-related marker in patients with HSCC, by using immunohistochemical technique as in our previously described tumor tissues [13].

\section{Patients and methods}

Patients. Between March 2000 and May 2012, seventy-eight patients with HSCC stage III or IV disease who were diagnosed as having HSCC, underwent surgical resection at Gunma University Hospital and Gunma Prefectural Cancer Center. Three patients without pathological specimens available and seven patients with pathological stage I or II disease were excluded from the current study. Thus, a total of 68 patients with stage III or IV were eligible for the study.

Formalin-fixed, paraffin-embedded primary tumor samples from the 68 patients were analyzed. All surgical specimens were reviewed and classified according to the WHO classification by an experienced pathologist who was unaware of the clinical or imaging findings. Pathologic tumor-node-metastasis (TNM) stages were established by using the Classification of Malignant Tumors provided by the International Union against Cancer (UICC) and American Joint Committee on Cancer (AJCC) system. The study protocol was approved by the institutional review board. The authors' approach to the evaluation and resection of these tumors has been described previously [13].

Immunohistochemical staining. GRP78/BiP was detected by using rabbit monoclonal antibodies (Cell Signaling Technology, Danvers, MA, 1:100 dilution). The detailed protocol for immunostaining has been published elsewhere $[14,15]$. GRP78/BiP expression scores were assessed according to the extent of staining as follows: $1, \leq 10 \%$ of tumor area stained; 2 , $11-25 \%$ stained; $3,26-50 \%$ stained; $4,51-75 \% ; 5, \geq 76 \%$ stained. The tumors in which stained tumor cells were scored as 3 to 5 were defined as high-expression tumors.

For CD34, Ki-67, and p53, immunohistochemical staining was performed according to the procedures described in a previous report $[14,15]$. The following antibodies were used: mouse monoclonal antibodies against CD34 (Nichirei, Tokyo, Japan; 1:800 dilution), Ki-67 (Dako, Glostrup, Denmark; 1:40 dilution), and p53 (D07; Dako; 1:50 dilution). The number of CD34-positive vessels was counted in four selected hotspots in a $400 \mathrm{X}$ field $\left(0.26 \mathrm{~mm}^{2}\right.$ field area). Microvessel density (MVD) was defined as the mean microvessel count per 0.26 $\mathrm{mm}^{2}$ field area. The median numbers of CD34-positive vessels were evaluated, and the tumors in which stained tumor cells exceeded the median value were defined as high-expression tumors. For Ki-67, a highly cellular area of the immunostained sections was evaluated. All epithelial cells with nuclear staining of any intensity were defined as high-expression cells. Approximately 1000 nuclei were counted on each slide. Proliferative activity was assessed as the percentage of Ki-67-stained nuclei (Ki-67 labeling index) in the sample. The median value of the Ki-67 labeling index was evaluated, and the tumors exceeding the median value were defined as high-expression tumors. For p53, a microscopic examination of the nuclear reaction product was performed and scored. Based on a previous report [14], p53 expression greater than $10 \%$ of the tumor cells was defined as positive expression. The sections were assessed by using light microscopy in a blinded fashion by at least two of the authors.

Statistical analysis. P-values of $<0.05$ indicated a statistically significant difference. Fisher's exact test was used to examine the association of two categorical variables. The correlation between different variables was analyzed by using the nonparametric Spearman's rank test. Kaplan-Meier method was used to estimate survival as a function of time, and survival differences were analyzed by using the log-rank test. Overall survival (OS) was determined as the time from tumor resection to death from any cause. Progression-free survival (PFS) was defined as the time between tumor resection and the first disease progression or death. Multivariate analyses were performed by using stepwise Cox proportional hazards model to identify independent prognostic factors. Statistical analyses were performed by using JMP 8 (SAS, Institute Inc., Cary, NC, USA) for Windows.

\section{Results}

Patient characteristics and immunohistochemical evaluation. The median age of the patients was 68 years (range, 37 to 82 years). Twelve patients had stage III disease, and 56 had stage IV disease. Forty-eight patients received postoperative adjuvant chemotherapy with cisplatin, 5-FU (fluorouracil), docetaxel, or S-1 (Taiho Pharmaceutical Co., Ltd, Tokyo, Japan), oral administration of tegafur (a fluorouracil derivative drug), and/or radiotherapy. None of the patients had received neo-adjuvant chemotherapy, and intraoperative therapy was also not performed on any patient. The day of surgery was considered as the starting day for measuring postoperative survival. The median follow-up time was 798 days (range, 43 to 3902 days).

The sixty-eight primary lesions were analyzed by using immunohistochemistry. Figure 1 reveals the representative imaging of GRP78/BiP. GRP78/BiP immunostaining was 
A

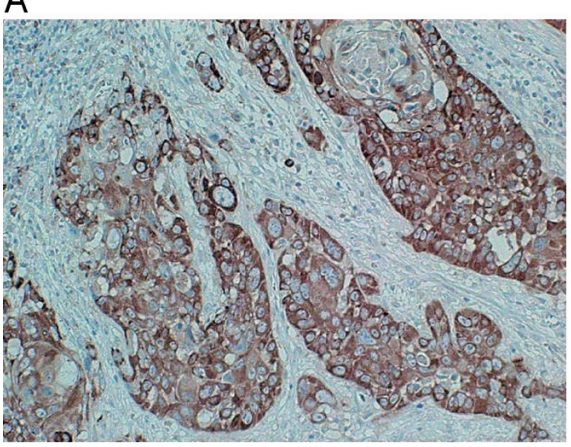

B

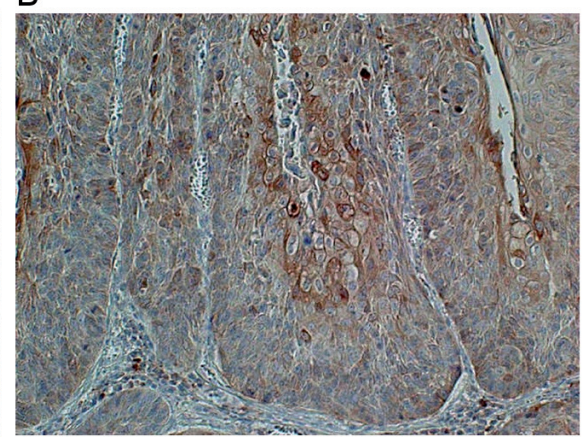

C

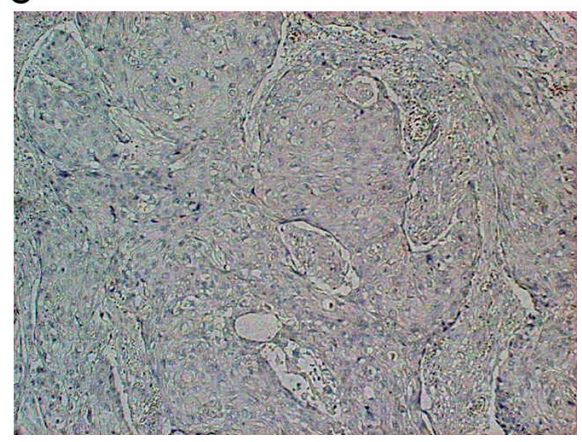

Figure 1. Immunohistochemical staining as representative imaging. Immunohistostaining of GRP78/BiP [high expression (scoring of 5) (A) and low expression (scoring of 2) (B)] showing the immunohistostaining pattern of cytoplasm or membrane. Figure 1C reveals negative staining for GRP78/BiP expression.

detected in the carcinoma cells in tumor tissues and was stained in the cytoplasm and membrane. Among 68 patients, the high expression rate of GRP78/BiP was observed in $80.8 \%$ (55/68). Figure 2 shows the percentage of GRP78/BiP expression according to the scoring scale of 1 to 5 . The percentages with GRP78/BiP scoring of $1,2,3,4$, and 5 were observed in $4 \%, 15 \%, 49 \%, 28 \%$, and $4 \%$ patients, respectively. We previously reported the results of immunohistochemical staining of Ki-67 and CD34 in HSCC [13]. The median number of CD34-positive vessels was 13 (range, 2-22), and this value was chosen as a cutoff point. The median value of the Ki-67 labeling index was 28 (range, 3-88), and the value of $28 \%$ was chosen as a cutoff point. Positive p53 expression was observed in $55.8 \%(38 / 68)$ patients.

Table 1 shows the patient demographics according to GRP78/BiP expression status. The high expression of GRP78/ $\mathrm{BiP}$ was not significantly associated with any variables, including previously reported data [13].

Table 1. Patient's demographics according to BIP/GPR78 expression

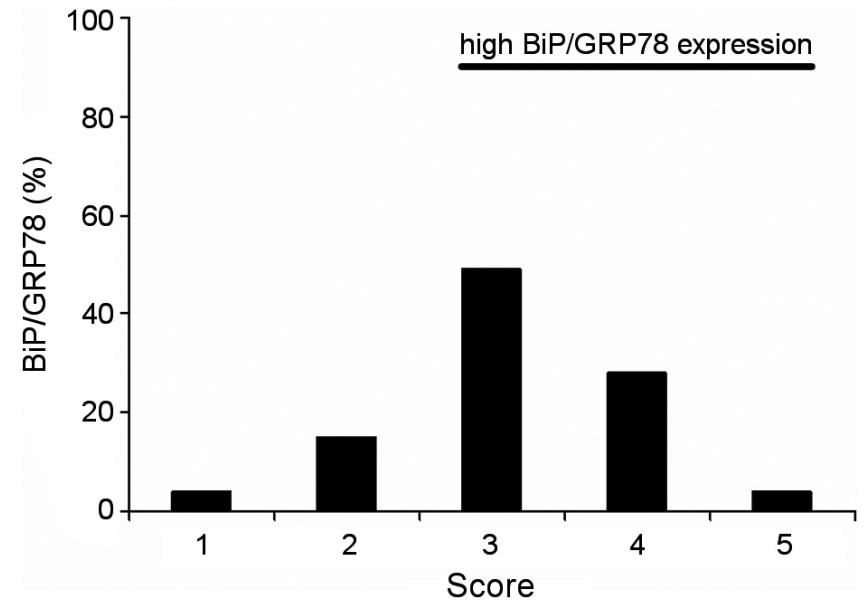

Figure 2. Percentage of GRP78/BiP expression according to scoring on a scale of 1 to 5 . The percentages with GRP78/BiP scoring of 1,2,3,4, and 5 were observed in $4 \%, 15 \%, 49 \%, 28 \%$, and $4 \%$, respectively.

\begin{tabular}{|c|c|c|c|c|c|}
\hline \multirow[b]{2}{*}{ Variables } & & \multirow{2}{*}{$\begin{array}{c}\text { Total } \\
(n=68) \\
\end{array}$} & \multicolumn{3}{|c|}{ BIP/GRP78 } \\
\hline & & & $\begin{array}{l}\text { High } \\
(n=55)\end{array}$ & $\frac{\text { Low }}{(n=13)}$ & $p$-value \\
\hline Age & $\leq 65 />65 \mathrm{yr}$ & $29 / 39$ & $23 / 32$ & $6 / 7$ & $>0.99$ \\
\hline Sex & Male / female & $52 / 16$ & $49 / 6$ & $10 / 3$ & 0.35 \\
\hline Tumor size (mm) & $\leq 35 />35 \mathrm{yr}$ & $25 / 43$ & $21 / 34$ & $4 / 9$ & 0.75 \\
\hline $\mathrm{T}$ factor & T1-2 / T3-4 & $15 / 53$ & $14 / 41$ & $1 / 12$ & 0.43 \\
\hline $\mathrm{N}$ factor & No / N1-2 & $13 / 55$ & $10 / 45$ & $3 / 10$ & 0.70 \\
\hline Disease stage & III / IV & $12 / 56$ & $9 / 46$ & $3 / 10$ & 0.68 \\
\hline Diferentiation & $\mathrm{WD} / \mathrm{MD}$ or $\mathrm{PD}$ & $22 / 46$ & $18 / 37$ & $4 / 9$ & $>0.99$ \\
\hline Ly & yes / No & $56 / 12$ & $45 / 10$ & $11 / 2$ & $>0.99$ \\
\hline $\mathbf{v}$ & High / Low & $46 / 22$ & $38 / 17$ & $8 / 5$ & 0.73 \\
\hline Resected status & Yes / No & $11 / 57$ & $10 / 45$ & $1 / 12$ & 0.68 \\
\hline Ki-67 & High / Low & $45 / 23$ & $38 / 17$ & $7 / 6$ & 0.33 \\
\hline CD34 & High / Low & $33 / 35$ & $29 / 26$ & $4 / 9$ & 0.05 \\
\hline p53 & Positive / Negative & $38 / 30$ & $31 / 24$ & $7 / 6$ & $>0.99$ \\
\hline
\end{tabular}

Abbreviation: WD, well differentiated; MD, moderate differentiated; PD, poorly differentiated; ly, lymphatic permeation; v, vascular invasion. 

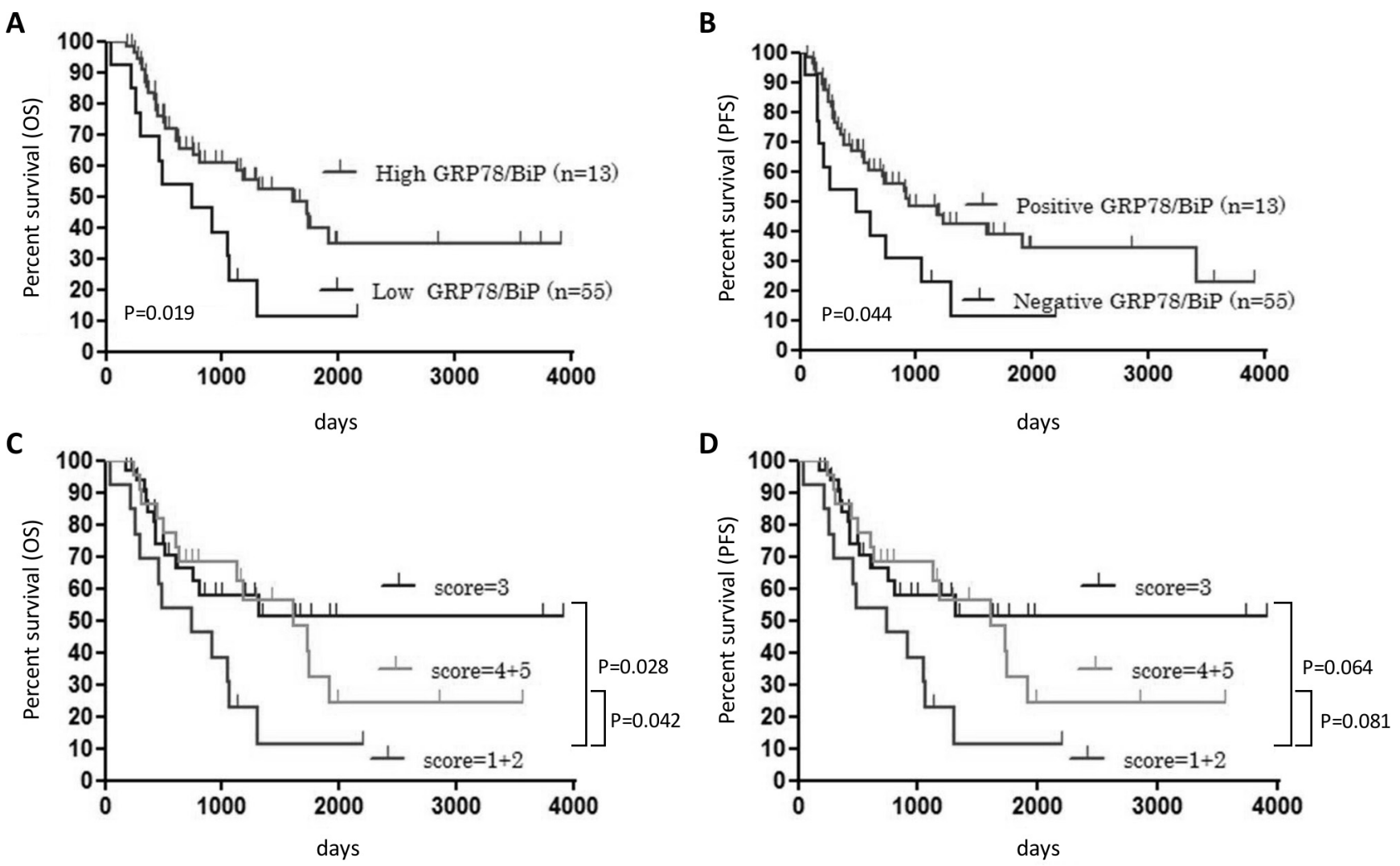

Figure 3. The Kaplan-Meier survival curve of overall survival (OS) and progression-free survival (PFS) according to the expression of GRP78/BiP: A statistically significant difference in OS and PFS was recognized between patients with high and low GRP78/BIP expression [OS, $p=0.019$ (A); PFS, $p=0.044$ (B)]. The Kaplan-Meier curve according to scoring of GRP78/BiP expression: A statistically significant difference in OS was observed between patients with scoring of $1+2$ and scoring of 3 ( $p=0.028$ ), and between scoring of $1+2$ and scoring of $4+5$ ( $p=0.042)(C)$. But, no statistically difference in PFS was recognized between patients with scoring of $1+2$ and scoring of 3 ( $p=0.064$ ), and between scoring of $1+2$ and scoring of $4+5$ $(\mathrm{p}=\mathbf{0 . 0 8 1})(\mathrm{D})$.

Correlation between GRP78/BiP expression and different variables. On using Spearman's rank correlation, GRP78/BiP had a statistically significant correlation with CD34 $(r=0.257, p=0.034)$, but not Ki-67 $(r=0.158 p=0.216)$ and CD34 $(r=-0.146, p=0.233)$ (Table 2).

Survival analysis. All patients showed a median survival time (MST) and 5-year survival rate for OS of 1127 days and $31.9 \%$, respectively; MST and 5-year survival rate for PFS were 898 days and $33.0 \%$, respectively. Figure 3 shows the

Table 2. Correlation with BIP/GRP78 expression

\begin{tabular}{lccc}
\hline & Spearman $\mathbf{r}$ & $\mathbf{9 5 \%} \mathbf{C I}$ & $\boldsymbol{p}$-value \\
\hline Tumor size & -0.146 & -0.378 to 0.102 & 0.233 \\
Ki-67 & 0.158 & -0.097 to 0.382 & 0.216 \\
CD34 & 0.257 & 0.012 to 0.473 & $\mathbf{0 . 0 3 4}$ \\
\hline
\end{tabular}

Abbreviation: 95\%CI, 95\% confidence interval.
Kaplan-Meier survival curve of OS and PFS according to the expression of GRP78/BiP. The univariate and multivariate analysis for all patients is listed in Table 3. On performing univariate analysis, lymphatic permeation, vascular invasion, and low GRP78/BiP expression were significantly associated with a shorter OS, and the significant prognostic variables for PFS were proven to be lymphatic permeation, vascular invasion, low GRP78/BiP expression, and positive p53. Multivariate analysis confirmed that the low expression of GRP78/ $\mathrm{BiP}$ was an independent factor for predicting poor OS and PFS after surgery.

Next, we performed the survival analysis according to scoring of GRP78/BiP expression (Fig. 3C and 3D). A statistically significant difference in the OS was observed between patients with scoring of $1+2$ and scoring of $3(\mathrm{p}=0.028)$, and between scoring of $1+2$ and scoring of $4+5$ ( $\mathrm{p}=0.042$ ) (Fig. 3C). However, no statistically difference in PFS was recognized between patients with scoring of $1+2$ and scoring of $3(\mathrm{p}=0.064)$, and between scoring of $1+2$ and scoring of $4+5$ $(\mathrm{p}=0.081)$ (Fig. 3D). 
Table 3. Univariable and multivariable survival analysis in all patients

\begin{tabular}{|c|c|c|c|c|c|c|c|c|c|c|c|}
\hline \multirow{3}{*}{ Variables } & & \multicolumn{5}{|c|}{ Overall survival } & \multicolumn{5}{|c|}{ Progression-free survival } \\
\hline & & \multicolumn{2}{|c|}{ Univariable } & \multicolumn{3}{|c|}{ Multivariable } & \multicolumn{2}{|c|}{ Univariable } & \multicolumn{3}{|c|}{ Multivariable } \\
\hline & & 5 -yrs rate(\%) & $P$-value & HR & $95 \% \mathrm{CI}$ & $P$-value & 5 -yrs rate(\%) & $P$-value & HR & $95 \% \mathrm{CI}$ & $P$-value \\
\hline Age & $\leq 65 />65 \mathrm{yr}$ & $45 / 17$ & 0.109 & & & & $36 / 27$ & 0.428 & & & \\
\hline Sex & Male / female & $35 / 27$ & 0.237 & & & & $33 / 26$ & 0.636 & & & \\
\hline Differentiation & $\mathrm{WD}$ or MD / PD & $34 / 31$ & 0.958 & & & & $31 / 32$ & 0.687 & & & \\
\hline Stage & III / IV & $64 / 28$ & 0.126 & 1.206 & $0.794-2.002$ & 0.399 & $37 / 26$ & 0.158 & 1.179 & $0.785-1.938$ & 0.448 \\
\hline Tumor size & $\leq 35 />35 \mathrm{yr}$ & $27 / 36$ & 0.411 & & & & $27 / 36$ & 0.222 & & & \\
\hline Lymphatic permeation & yes / no & $24 / 77$ & 0.002 & 1.847 & 0.794-4.705 & 0.066 & $27 / 53$ & 0.048 & 1.258 & $0.739-2.419$ & 0.420 \\
\hline Vascular invasion & yes / no & $27 / 63$ & 0.032 & 1.354 & $0.897-2.191$ & 0.155 & $23 / 62$ & 0.007 & 1.361 & $1.021-2.575$ & 0.038 \\
\hline Resected status & yes / no & $26 / 35$ & 0.327 & & & & $21 / 34$ & 0.225 & & & \\
\hline GRP78/BIP & High / Low & $37 / 11$ & 0.019 & 1.549 & $1.063-2.175$ & 0.024 & $33 / 11$ & 0.044 & 1.492 & $1.031-2.083$ & 0.035 \\
\hline CD34 & High / Low & $16 / 44$ & 0.269 & & & & $18 / 45$ & 0.069 & & & \\
\hline $\mathrm{Ki}-67$ & High / Low & $32 / 38$ & 0.655 & & & & $33 / 32$ & 0.999 & & & \\
\hline p53 & Positive/Negative & $27 / 32$ & 0.271 & & & & $20 / 49$ & 0.017 & & & \\
\hline
\end{tabular}

Abbreviation: WD, well differentiated; MD, moderate differentiated; PD, poorly differentiated; HR, hazard ratio; $95 \%$ CI, 95\% confidence interval.

\section{Discussion}

To our knowledge, this is the first study investigating the prognostic significance of GRP78/BiP expression in patients with advanced HSCC. We found that the decreasing protein expression of GRP78/BiP is closely associated with worse survival after surgery. The expression level of GRP78/BiP was confirmed as an independent prognostic predictor by performing multivariate analysis. The presented study focused on the patients with advanced disease (stage III or IV); therefore, no statistically significant difference in the variables indicating tumor aggressiveness and cell proliferation was observed between patients with high and low GRP78/BiP expression. The results of our study suggest that the reduced expression of GRP78/BiP is important as a negative marker for predicting worse prognosis in the patients with advanced HSCC who underwent surgical resection.

Recently, we found a contrary discussion about the prognostic significance of GRP78/BiP expression level in various human cancers. The increasing GRP78/BiP expression predicted a worse prognosis than the decreasing expression in patients with hepatocellular carcinoma, gastric cancer, prostate cancer, and renal cell carcinoma $[5,6,10-12]$. On the other hand, the low expression of GRP78/BiP in patients with oral squamous cell carcinoma and lung cancer predicted a poorer outcome than did high expression [7, 9]. Huang et al. showed that decreased GRP78/BiP expression is closely associated with advanced tumor stage and lymph node metastasis in patients with oral SCC in Taiwan [7]. On the other hand, Xia et al. depicted that the elevation of GRP78/BiP expression was exhibited as a negative prognostic factor in patients with oral SCC and was closely related to the tumor aggressiveness such as metastasis, advanced disease staging, and lymphatic invasion [5]. The mechanism of these opposite results remains unclear; however, the results of this study demonstrated that high-grade cancer cells may overcome ER stress-induced apoptosis by reducing ER stress response, whereas low-grade cancer cells are under ER stress conditions, which can be protective for tumor cells. Furthermore, the prognostic role of ER stress among head and neck cancers may vary according to the primary site location and its disease staging. Further study is warranted to elucidate the significant difference of GRP78/BiP expression between the patients with early and advanced disease stage.

GRP78/BiP is a characterized ER chaperone and is part of the Hsp70 family [16]. The cytoprotective and antiapoptotic function of GRP78/BiP has been known to lead to the modulation of chemosensitivity [3]. In an experimental study, it was reported that silencing of GRP78/BiP expression may be related to the loss of PTEN tumor suppression and oncogenic AKT activation [17]. A recent review showed that the elevated expression of GRP78/BiP significantly correlates with worse survival, tumor recurrence, and chemo-resistance [3]. Although the down-regulation or inhibition of GRP78/BiP alternation may have the potential to be a molecular target for the therapeutics of several cancers, it has also been shown that the induction of GRP78/BiP activity could be a novel target against the drug resistant cells of lung, breast, and bladder cancers $[4,12,16]$. It remains unclear whether the induction or inhibition of GRP78/BiP is effective as a potential of targeting therapy. The mechanism for targeting GRP78/BiP on tumor progression, development and survival should be elucidated by further studies.

There are several limitations of the current study. First, the sample size was small, which may bias the results of our study. The HSCC patients with advanced disease, like those included in our study, are candidates for initial surgical resection, whereas chemo-radiotherapy without surgical resection 
is generally recommended as a standard treatment against those with early disease such as stage I or II. Since it is impossible to obtain adequate specimens from HSCC patients with early disease and the immunohistochemical analysis is limited, such patients were excluded from the present study. Therefore, our study focused on the investigation of the patients with advanced disease who underwent surgical resection. Second, the antibody of GRP78/BiP used in our study was different from that (Santa Cruz Biotechnology, sc-13968) used in the previous studies examining oral SCC $[5,7]$. We assessed the quality of GRP78/BiP among some antibodies and we believe that the antibody we used is best suited to evaluate the protein expression of GRP78/BiP. Further study is warranted to investigate the prognostic role of GRP78/BiP expression in human neoplasms by utilization of the same antibody and immunohistochemical technique. Finally, we could not clarify the reason why the decreased expression of GRP78/BiP is closely associated with shorter survival for HSCC patients with advanced disease. The results of our study are partially similar to those obtained in previous studies, but it remains unclear why the prognostic role of ER stress marker varies according to the degree of GRP78/BiP expression.

In conclusion, the decreasing expression of GRP78/BiP, ER stress marker was identified as a significant predictor related to shorter survival after surgery for treating advanced HSCC. Our study suggests that the reduced expression of GRP78/BiP contributes to negative survival for head and neck cancers with advanced disease, and the induction of GRP78/BiP could have potential to prognosticate this condition.

Acknowledgements: We appreciate Ms. Yuka Matsui for her technical assistance of manuscript submission and Ms. Tomoko Okada for her data collection and technical assistance. We also deeply appreciate Prof. Masahiko Nishiyama of Department of Molecular Pharmacology and Oncology, Gunma University Graduate School of Medicine, for the critical review of this manuscript. This work was supported in part by Grant 23591750 (K. K) and Grant 23592523 (K. C) from the Ministry of Education, Culture, Sports, Science and Technology, Japan, and National Hospital Organization Policy Based Medical Services. We thank all staff members of the Department of Pathology and Clinical Laboratories at Gunma Prefectural Cancer Center for their technical assistance in the immunohistochemical analysis.

\section{References}

[1] RAGIN CC, MODUGNO F, GOLLIN SM. The epidemiology and risk factors of head and neck cancer: a focus on human papillomavirus. J Dent Res. 2007;86:104-114. http://dx.doi. org/10.1177/154405910708600202

[2] HENDERSHOT LM. The ER function BiP is a master regulator of ER function. Mt Sinai J Med 2004; 71: 289-297.

[3] LI J, LEE AS. Stress induction of GRP78/BiP and its role in cancer. Curr Mol Med 2006; 6: 45-54. http://dx.doi. org/10.2174/156652406775574523
[4] GAZIT G, LU J, LEE AS. De-regulation of GRP stress protein expression in human breast cancer cell lines. Breast Cancer Res Treat 1999; 54: 135-146. http://dx.doi. org/10.1023/A:1006102411439

[5] XIA F, XU JC, ZHANG P, ZHANG YY, ZHANG QW, et al. Glusose-rekated protein 78 and heparanase expression in oral squamous cell carcinoma: correlation and prognostic significance. World J Surg Oncol 2014;12:121. http://dx.doi. org/10.1186/1477-7819-12-121

[6] LIN CY, CHEN WH, LIAO CT, CHEN IH, CHIU CC, et al. Positive association of glucose-related protein 78 during oral cancer progression and the prognostic value in oral precancerous lesions. Head Neck 2010;32:1028-1039. http://dx.doi. org/10.1002/hed.21287

[7] HUANG TT, CHEN JY, TSENG CE, SU YC, HO HC, et al. Decreased GRP78 protein expression is a potential prognostic marker of oral squamous cell carcinoma in Taiwan. J Formos Med Assoc. 2010;109:326-337. http://dx.doi.org/10.1016/ $\underline{\text { S0929-6646(10)60060-5 }}$

[8] VANDEWYNCKEL YP, LAUKENS D, BOGAERTS E, VANHOVE C, DESCAMPS B, et al. Modulation of the unfolded protein response impedes tumor cell adaptation to proteotoxic stress: a PERK for hepatocellular carcinoma therapy. Hepatol Int 2015;9:93-104. http://dx.doi.org/10.1007/s12072-014-9582-0

[9] URAMOTO H, SUGIO K, OYAMA T, NAKATA S, ONO K, et al. Expression of endoplasmic reticulum molecular chaperone Grp78 in human lung cancer and its clinical significance. Lung Cancer 2005;49:55-62. http://dx.doi.org/10.1016/j. lungcan.2004.12.011

[10] ZHANG J, JIANG Y, JIA Z, LI Q, GONG W, et al. Association of elevated GRP78 expression with increased lymph node metastasis and poor prognosis in patients with gastric cancer. Clin Exp Metastasis 2006;23:401-410. http://dx.doi. org/10.1007/s10585-006-9051-9

[11] DANESHMAND S, QUEK ML, LIN E, LEE C, COTE RJ, et al. Glucose-regulated protein GRP78 is up-regulated in prostate cancer and correlates with recurrence and survival. Hum Pathol 2007;38:1547-1552. http://dx.doi.org/10.1016/j. humpath.2007.03.014

[12] FU W, WU X, LI J, MO Z, YANG Z, et al. Upregulation of GRP78 in renal cell carcinoma and its significance. Urology 2010;75:603-607. http://dx.doi.org/10.1016/j. urology.2009.05.007

[13] TOYODA M, KAIRA K, SHINO M, SAKAKURA K, TAKAHASHI K, et al. CD98 is a novel prognostic indicator for patients with stage III/IV hypopharyngeal squamous cell carcinoma. Head Neck. 2015;37:1569-1574. http://dx.doi. org/10.1002/hed.23797

[14] TOYODA M, KAIRA K, OHSHIMA Y, ISHIOKA NS, SHINO M, et al. Prognostic significance of Amino Acid Transporter Expression (LAT1, ASCT2 and xCT) in Surgically Resected Tongue Cancer. Br J Cancer 2014;110:2506-13. http://dx.doi.org/10.1038/bjc.2014.178

[15] KAIRA K, TOYODA M, SHIMIZU A, SHINO M, SAKAKURA K, et al. Expression of ER stress markers (GRP78/BiP and PERK) in adenoid cystic carcinoma. Acta Oto-laryngologica 2015;14:1-7. 
[16] WANG Q, HE Z, ZHANG J, WANG Y, WANG T, et al. Overexpression of endoplasmic reticulum molecular chaperone GRP94 and GRP78 in human lung cancer tissues and its significance. Cancer Detect Prev 2005;29:544-551. http:// dx.doi.org/10.1016/j.cdp.2005.09.010
[17] FU Y, WEY S, WANG M, YE R, LIAO CP, et al. Pten null prostate tumorigenesis and AKT activation are blocked by targeted knockout of ER chaperone GRP78/BiP in prostate epithelium. Proc Natl Acad Sci USA 2008;105:19444-449. http://dx.doi.org/10.1073/pnas.0807691105 